\title{
L'HOMME L'Homme
}

Revue française d'anthropologie

157 | janvier-mars 2001

Représentations et temporalités

\section{Gabriele Stürzenhofecker, Times Enmeshed. Gender, Space, and History among the Duna of Papua New}

Guinea

Stanford, Ca, Stanford University Press, 1998, XVIII + 242 p., append., bibl., index, tabl., ph.

Pascale Bonnemère

\section{CpenEdition}

Journals

Édition électronique

URL : http://journals.openedition.org//homme/5896

DOI : 10.4000//homme.5896

ISSN : 1953-8103

Éditeur

Éditions de l'EHESS

Édition imprimée

Date de publication : 1 janvier 2001

Pagination : 322-325

ISBN : 2-7132-1357-6

ISSN : 0439-4216

Référence électronique

Pascale Bonnemère, « Gabriele Stürzenhofecker, Times Enmeshed. Gender, Space, and History among the Duna of Papua New Guinea », L'Homme [En ligne], 157 | janvier-mars 2001, mis en ligne le 23 mai 2007, consulté le 24 septembre 2020. URL : http://journals.openedition.org//homme/5896 ; DOI : https://doi.org/10.4000//homme.5896

Ce document a été généré automatiquement le 24 septembre 2020.

(c) École des hautes études en sciences sociales 


\section{Gabriele Stürzenhofecker, Times Enmeshed. Gender, Space, and History among the Duna of Papua New Guinea}

Stanford, Ca, Stanford University Press, 1998, XVIII + 242 p., append., bibl., index, tabl., ph.

Pascale Bonnemère

1 DANS CE LIVRE consacré aux Duna de la vallée d'Aluni, en Papouasie Nouvelle-Guinée, Gabriele Stürzenhofecker propose une analyse des matériaux ethnographiques qu'elle a recueillis entre 1991 et 1994, en prenant pour axes principaux de sa réflexion la construction socioculturelle de la différence entre les sexes («gender as an idiom of thinking » ; p. 8) et la perception locale du temps et de l'histoire.

2 Deux préoccupations majeures parcourent donc cet ouvrage. Il s'agit, d'une part, de s'interroger sur la conscience qu'ont les Duna de leur histoire et d'évaluer les bouleversements que leur vision du cosmos et de sa reproduction a subis avec l'arrivée des missionnaires, dépositaires d'une perception temporelle différente; d'autre part, d'analyser les difficultés actuelles ressenties par les hommes dans leurs relations avec les femmes et, partant, dans la définition de leur propre identité. De cette étude approfondie, il ressort que la sorcellerie et les modes de consommation et de partage de la nourriture constituent des espaces sociaux où s'expriment avec force les tensions nées des formes nouvelles que prennent les relations entre les hommes et les femmes. Par suite du déclin des rituels masculins notamment, les hommes ont perdu une bonne part du contrôle qu'ils exerçaient sur les activités féminines, et des zones d'ambiguïté et d'incertitude sont apparues où ni les hommes ni les femmes ne semblent trouver leur compte.

3 Pour parler des transformations dont leur vie a été témoin, les Duna emploient une métaphore qui fait référence à la terre. Dans toute cette région, le sol a une importance cosmologique fondamentale : il est le moyen par lequel les gens conçoivent le passage du temps et créent leur identité. Sous une manifestation matérielle unique, il englobe l'espace, le temps et l'histoire (p. 74). 
4 Le sol obéit à un rythme de vie cyclique et son renouvellement était autrefois ponctué par des rituels de fertilité destinés à Payeme Ima, esprit féminin polymorphe assurant la prospérité pour peu que l'on ait effectué les sacrifices requis. Le spectre d'une possible modification, voire d'un épuisement du sol, a toujours existé dans la conscience des Duna qui, encore aujourd'hui, accordent une grande attention à la lecture du sol en notant les changements (de végétation surtout) survenus et en essayant de les interpréter. Cependant, s'ils disposaient autrefois des moyens rituels appropriés pour prévenir la « fin du sol » (the ending of the ground), tel n'est plus le cas de nos jours, ce qui donne au discours millénariste de l'Église apostolique d'Aluni une consonance dramatique, puisqu'il vient renforcer leur anxiété de ne plus disposer des moyens de régénérer la terre.

5 Selon l'auteur, cette vision cyclique du cosmos matérialisée dans l'état du sol trouve un écho dans le système des paiements matrimoniaux qui donnent lieu à un cycle d'endettement se reproduisant de génération en génération. Un homme ne s'affranchit des dettes qu'il a contractées à son mariage qu'à celui de sa propre fille, si bien que la fermeture d'un cycle d'endettement particulier centré sur une femme dépend de l'ouverture d'un autre cycle centré sur la fille de celle-ci. Mais le système des paiements matrimoniaux n'est qu'une partie d'une logique culturelle plus vaste qui sous-tend les notions duna de temporalité et de cosmologie (p. 121). Il est aussi l'institution à travers laquelle les hommes exercent une forme de contrôle sur l'action productive et reproductrice des femmes (p.192), et donc le lieu où se maintiennent les inégalités entre les sexes (p.100). Les femmes sont liées à ce système de bridewealth non seulement parce qu'elles assurent la reproduction des êtres humains, mais aussi parce que ce sont elles qui produisent les biens servant à éteindre les dettes contractées lorsque leurs maris les ont épousées. Cela dit, pour plusieurs raisons, dont l'absence d'échanges cérémoniels et même de prestations fondées sur les liens d'affinité (p. 105), cette position n'est guère comparable à celle des femmes melpa, par exemple, dont on connaît bien le statut particulier («in-between ») et le rôle qu'elles ont de ce fait dans le déroulement de ces échanges ${ }^{1}$.

6 Le contrôle qu'exerçaient les hommes sur leurs épouses s'est aussi émoussé dans d'autres contextes. La nourriture et son partage avaient toujours constitué une sphère où la séparation entre les sexes était nettement marquée. Avant la colonisation, il n'y avait que peu de partage de nourriture entre hommes et femmes: non seulement aucune obligation n'incombait aux épouses de nourrir leur mari, mais, pour ce qui était des aliments prisés comme le porc, les adultes se constituaient en groupes d'individus de même sexe, les hommes et les femmes consommant des portions différentes de l'animal (p. 136). Dans la mesure où il existe une forte association entre la personne et la consommation de nourriture, les changements qui ont eu pour effet d'offrir aux femmes un accès à des morceaux qui leur étaient auparavant interdits affectent profondément l'identité masculine. Il est probable que l'abandon de leurs activités rituelles a généré une plus grande anxiété chez les hommes, dont l'identité personnelle et collective était précisément fondée sur un type spécifique de consommation (p. 191), lié en particulier à la rupture qu'il leur fallait établir avec la période de l'enfance, en recevant leur nourriture non plus des mains de leur mère mais d'experts rituels masculins, ce qui était considéré comme une étape très importante de cette nouvelle socialisation opérée dans et par le rituel. De surcroit, la séparation d'avec la mère et la nourriture maternelle allait de pair avec l'apprentissage de la morale sexuelle. On a 
donc ici un rapport étroit entre la personne, les règles de consommation et les comportements moralement adéquats (p.171), qui explique pourquoi l'abandon des cultes masculins et les changements affectant le partage et l'usage de nourriture dans la société ont retenti sur la perception et la constitution de l'individu, en particulier en ce qui concerne les hommes et leurs relations avec les femmes.

7 À cette fragilisation de l'identité masculine s'ajoute l'imagerie associée aux agents de la sorcellerie, majoritairement des femmes, créditées par les hommes d'une avidité hors du commun pour la nourriture en général et la viande en particulier (pp. 168-169). Dans le passé, les sorcières représentaient une pièce essentielle des relations avec les groupes ennemis et leurs pouvoirs étaient parfois utilisés comme armes de guerre, ce qui permettait de diriger leur malveillance vers l'extérieur plutôt que de les laisser agir au sein du groupe (p.182). Aujourd'hui, ces agents de la sorcellerie s'attaquent à la communauté villageoise, détruisant sa force de l'intérieur. Le texte n'indique malheureusement pas si ces femmes dotées de pouvoirs malveillants proviennent toujours de groupes anciennement ennemis, ou si certaines peuvent être d'origine duna.

8 Il n'en reste pas moins que, par les caractères qu'elle revêt (avidité, dévoration des organes vitaux internes, association de la sorcellerie à la féminité adulte, impossibilité de guérison sans intervention du sorcier, mobilité extrême de celui-ci, transmission des pouvoirs malveillants en ligne utérine), la sorcellerie condense une grande partie des problèmes nés de la perte de contrôle des comportements féminins par les hommes, consécutive à l'arrivée des missionnaires et aux altérations de la vie sociale qu'ils ont imposées. L'évolution des pratiques divinatoires illustre parfaitement ce phénomène : alors que le repérage des sorciers était jadis une prérogative masculine, leur identification résulte aujourd'hui de dénonciations par des sorcières repenties, ou d'auto-confessions.

9 On pourrait penser que, de cette situation, les hommes pâtissent finalement plus que les femmes, lesquelles ont vu s'élargir leur accès à l'espace communal et à la nourriture. Les formes que revêt aujourd'hui la sorcellerie montrent qu'il n'en est rien, puisqu'elles restent les principales accusées, et le sont même de plus en plus. Ainsi, les Duna se trouvent désormais dans une situation extrême - c'est du moins l'impression qui ressort de cet ouvrage -, où les hommes craignent les actions malveillantes des femmes en âge de procréer et où celles-ci ont constamment peur d'être accusées en cas de décès dans la communauté.

10 Times Enmeshed est construit d'une manière analytique plutôt que descriptive, ce qui constitue à la fois un avantage et un inconvénient. L'organisation choisie est un des points forts du livre en ce qu'elle permet notamment de le distinguer des monographies plus classiques, que - à tort ou à raison - l'anthropologie contemporaine a tendance à dénigrer ; mais, de ce fait, il n'est pas d'une lecture aisée, même pour les spécialistes.

11 Alors que Gabriele Stürzenhofecker situe, encore que discrètement, son travail dans le courant de l'anthropologie historique de l'Océanie (pp. 194, 200), une des conclusions auxquelles aboutit son analyse semble aller à l'encontre de certaines positions défendues par cette branche de la discipline. En effet, l'auteur note à plusieurs reprises à quel point les formes de pensée des Duna ont persisté malgré les profonds changements survenus dans les pratiques. Outre son intérêt ethnographique, cet ouvrage a donc, à mon sens, le mérite de révéler un tel phénomène, probablement plus 
répandu qu'on ne le croit, au moins chez des groupes qui n'ont rencontré des étrangers qu'à partir des années 30 , comme c'est le cas dans certaines zones de l'intérieur de la Nouvelle-Guinée. Là résident peut-être les difficultés et les tensions qui existent dans la société duna actuelle et que l'auteur met parfaitement en lumière.

L'ouvrage de Gabriele Stürzenhofecker présente quelques petits défauts dont le principal est de laisser parfois au lecteur le soin de comprendre par lui-même les implications des arguments avancés; en bref, elle s'arrête un peu trop vite en chemin ou ne fournit pas tous les éléments nécessaires. Il est par exemple dommage qu'elle n'ait pas développé davantage le concept d'" internal externality " qui renvoie aux règles implicites d'un système matrimonial où les descendants de mariages entre Duna d'Aluni et groupes voisins Yeru et Bogaia n'adoptent pas les mêmes stratégies de mariage selon qu'ils sont homme ou femme. Il eut été intéressant d'analyser plus avant un tel phénomène, d'autant qu'il opère aussi en sorcellerie.

Ces remarques formulées, il n'en reste pas moins que ce premier livre est des plus solides. Il cumule et croise pour le meilleur des données ethnographiques aussi bien qu'historiques, et cherche toujours à privilégier une vision dite " émique » de la réalité, en particulier des bouleversements survenus dans un passé récent (p. 193). Il fournit des informations précieuses sur la sorcellerie, la nourriture et les formes du " gender " chez les Duna, tout en donnant matière à réfléchir plus généralement sur l'histoire, sur la nature des transformations qu'elle opère et, partant, sur la structure. Il est rare de trouver tous ces éléments réunis au sein d'un même ouvrage.

\section{NOTES}

1. Cf. Marilyn Strathern, Women in Between. Females Roles in a Male World, Mount Hagen, New Guinea, London-New York, Seminar Press, 1972 ( « Seminar Studies in Anthropology » 2).

\section{AUTEUR}

\section{PASCALE BONNEMÈRE}

CNRS, Centre de recherche et de documentation sur l'Océanie (CREDO), Marseille. 